\title{
Effect of Grain Size on High Frequency Loss in M-Type Hexaferrites $\mathrm{BaFe}_{12-x}\left(\mathrm{Ti}_{0.5} \mathrm{Mn}_{0.5}\right)_{x} \mathrm{O}_{19}(0 \leq x \leq 5)$ \\ A. HARADA ${ }^{\text {a) }}$, T. ISHIKURA ${ }^{\text {a) }}$, M. MURAMOTO ${ }^{\text {b) }}$, K. YASUHARA ${ }^{\text {a) }}$, and S. ITOH ${ }^{\text {a) }}$
}

a) Materials \& Prosess Development Center, TDK Corporation, 570-2, Matsugashita, Minami-Hatori, Narita, Chiba 286-8588, Japan

b) Devices Development Center, TDK Corporation, 2-15-7, Higashi-Oowada, Ichikawa, Chiba 272-8558, Japan

\begin{abstract}
Effect of $\mathrm{Fe}^{3+}$ substitution and grain size on magnetic properties in $\mathrm{GHz}$ range of M-type hexaferrites $\mathrm{BaFe}_{12-\mathrm{x}}\left(\mathrm{Ti}_{0.5} \mathrm{Mn}_{0.5}\right)_{\mathrm{x}} \mathrm{O}_{19}(0 \leq \mathrm{x} \leq$ 5) was investigated. Measured complex permeability spectra were well fitted by a model consisting of two types of magnetic susceptibilities corresponding to gyromagnetic spin rotation and domain wall motion. The model fitting shows $\mu^{\prime}$ in the GHz range is increased when a contribution of the gyromagnetic spin rotation is enhanced. Furthermore, from grain size distribution, it is shown that magnetic loss in the $\mathrm{GHz}$ range could be reduced when the average grain size increases and coefficient of variation (CV) decreases. This indicates contribution of a domain wall motion to the magnetic loss is reduced because the domain wall resonance peak shifts to lower frequency and becomes narrower. In our study, it is demonstrated that enhancement of $\mu^{\prime}$ with low magnetic loss is possible in the GHz region between two resonance peaks of a lower domain wall peak and a higher gyromagnetic spin rotation peak in M-type hexaferrites.
\end{abstract}

Key Words: M-type hexaferrite, High frequency, Complex permeability, Grain size, Magnetic loss

\section{INTRODUCTION}

Frequency band used for recent wireless devices has become higher. For example, a GPS chip mounted on a smartphone is used at $1.57 \mathrm{GHz}$. On the other hand, size of the devices has become smaller. Therefore, electronic components such as antennas and inductors used in these devices are also required to cope with both high frequency range availability and down sizing. In order to resolve this problem, application of a magnetic material with both low magnetic loss and high magnetic permeability at the high frequencies to the devices is suggested [1]. As an example of magnetic material performed in high frequency region, there is an M-type hexaferrites possessing high natural resonance frequency beyond the Snoek's limit because of the large anisotropy field $H_{\mathrm{A}}$ along c-axis of a hexagonal lattice. There are a number of studies on M-type hexaferrites as a soft magnetic material for magnetic recording media [2], microwave absorbers [3], etc. Recent years, application of an M-type hexaferrite for antennas used in GHz range is reported [4]. However, adequate radiation efficiency has not yet been acquired because of high magnetic loss. To develop a magnetic material with low magnetic loss and high $\mu^{\prime}$ in high frequency region, we investigated the effect of $\mathrm{Fe}^{3+}$ substitution and grain size on magnetic properties of M-Type hexaferrites $\mathrm{BaFe}_{12-x}\left(\mathrm{Ti}_{0.5} \mathrm{Mn}_{0.5}\right)_{x} \mathrm{O}_{19}(0 \leq x \leq 5)$ in $\mathrm{GHz}$ range.

\section{EXPERIMENTAL}

M-type hexaferrites $\mathrm{BaFe}_{12-x}\left(\mathrm{Ti}_{0.5} \mathrm{Mn}_{0.5}\right)_{x} \mathrm{O}_{19}(0 \leq x \leq 5)$ were prepared by conventional solid-state reaction. $\mathrm{Fe}_{2} \mathrm{O}_{3}, \mathrm{BaCO}_{3}$, $\mathrm{Mn}_{3} \mathrm{O}_{4}$, and $\mathrm{TiO}_{2}$ were mixed with water using ball milling. The mixture was calcined at $1473 \mathrm{~K}$ in air. A powder calcined was pulverized using dry vibratory rod milling and ball milling. The milled powder was sintered for $2 \mathrm{~h}$ separately at 1523 , 1573 , or $1623 \mathrm{~K}$ in $\mathrm{O}_{2}$. Complex permeability was measured as S-parameter using an impedance analyzer $(10 \mathrm{MHz}-1$ $\mathrm{GHz})$ and a network analyzer $(1 \mathrm{GHz}-15 \mathrm{GHz})$. Anisotropy field $H_{\mathrm{A}}$ was estimated from magnetization curves measured using a vibrating sample magnetometer (VSM). Grain size was measured from scanning electron microscope (SEM) images.

\section{RESULTS AND DISCUSSION}

Figure 1 shows real part $\mu^{\prime}$ and imaginary part $\mu^{\prime \prime}$ of complex permeability spectra of $\mathrm{BaFe}_{12-x}\left(\mathrm{Ti}_{0.5} \mathrm{Mn}_{0.5}\right)_{x} \mathrm{O}_{19}(0 \leq x \leq 5)$ sintered at $1523 \mathrm{~K}$. With increase of $x$, a resonance peak of $\mu^{\prime \prime}$ in GHz range shifts to lower frequency and $\mu^{\prime}$ increases at frequency lower than the resonance peak. Here, natural resonance frequency $f_{\mathrm{r}}$ and initial permeability $\mu_{\mathrm{i}}$ associated with spin rotation are expressed by the following formula, $f_{\mathrm{r}}=\gamma H_{\mathrm{A}} /(2 \pi)$ and $\mu_{\mathrm{i}}=2 M_{\mathrm{S}} /\left(3 \mu_{0} H_{\mathrm{A}}\right)+1$, where $\gamma$ is gyromagnetic constant and $M_{\mathrm{S}}$ is saturation magnetization. Since $H_{\mathrm{A}}$ decreased with increase of $x$ (Table I), it is thought that the increase in $\mu^{\prime}$ and the resonance peak shift in $\mu^{\prime \prime}$ are related to spin rotation. 


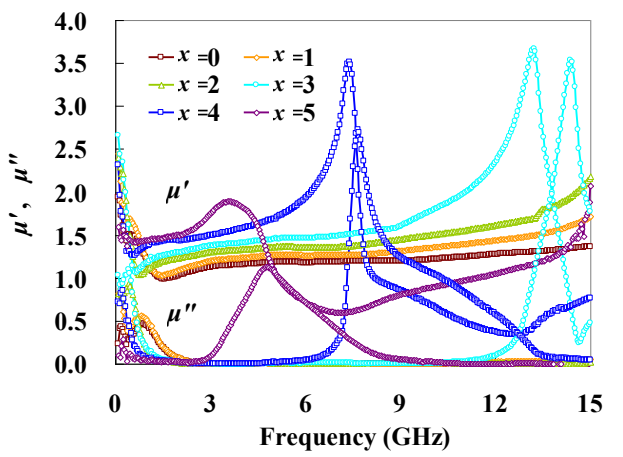

Table I Anisotropy field $H_{\mathrm{A}}$ estimated from $M H$ curve of $\mathrm{BaFe}_{12-x}\left(\mathrm{Ti}_{0.5} \mathrm{Mn}_{0.5}\right)_{x} \mathrm{O}_{19}$.

\begin{tabular}{cc}
\hline \hline $\boldsymbol{x}$ & $\boldsymbol{H}_{\mathrm{A}}(\mathrm{kOe})$ \\
\hline 0 & 13.6 \\
1 & 10.8 \\
2 & 9.3 \\
3 & 9.3 \\
4 & 8.4 \\
\hline \hline
\end{tabular}

Fig. 1 Real part $\mu^{\prime}$ and imaginary part $\mu^{\prime \prime}$ of complex permeability spectra of $\mathrm{BaFe}_{12-\mathrm{x}}\left(\mathrm{Ti}_{0.5} \mathrm{Mn}_{0.5}\right)_{\mathrm{x}} \mathrm{O}_{19}$ with different substitution contents.

To investigate influence of both spin rotation and domain wall motion on complex permeability, a fitting model is applied to the measured complex permeability spectra of $\mathrm{BaFe}_{8}\left(\mathrm{Ti}_{0.5} \mathrm{Mn}_{0.5}\right)_{4} \mathrm{O}_{19}$ sintered separately at 1523,1573 , or $1623 \mathrm{~K}$. The model for frequency-dependent magnetic permeability $\mu(\omega)$ consists of two types of magnetic susceptibilities. One is $\chi_{\mathrm{s}}(\omega)$ corresponding to gyromagnetic spin rotation and the other is $\chi_{\mathrm{d}}(\omega)$ corresponding to domain wall motion [5],

$$
\mu(\omega)=1+\chi_{s}(\omega)+\chi_{d}(\omega) \quad(1), \quad \chi_{s}(\omega)=\frac{\left(\omega_{s}+i \omega \alpha\right) \chi_{s 0} \omega_{s}}{\left(\omega_{s}+i \omega \alpha\right)^{2}-\omega^{2}} \quad(2), \quad \chi_{d}(\omega)=\frac{\chi_{d 0} \omega_{d}{ }^{2}}{\omega_{d}{ }^{2}-\omega^{2}+i \omega \beta}
$$

where $\chi_{\mathrm{s} 0}$ and $\chi_{\mathrm{d} 0}$ are static magnetic susceptibilities, $\omega_{\mathrm{s}}$ and $\omega_{\mathrm{d}}$ are resonance frequencies, $\alpha$ and $\beta$ are damping constants corresponding to spin and domain wall motion, respectively. The result of model fitting is shown in Figure 2 and Table II. Calculated curves are consistent with measured curves of $\mathrm{BaFe}_{8}\left(\mathrm{Ti}_{0.5} \mathrm{Mn}_{0.5}\right)_{4} \mathrm{O}_{19}$ very well. A measured value of $\mu^{\prime}$ at 1.57 $\mathrm{GHz}$ is increased from 1.42 to 1.52 according to sintering temperature from $1523 \mathrm{~K}$ to $1623 \mathrm{~K}$. On the other hand, when the fitting is applied to the spectra of 1523,1573 , and $1623 \mathrm{~K}$, although $\chi_{\mathrm{d} 0}$ decreases from 1.40 to $0.81, \chi_{\mathrm{s} 0}$ increased from 0.45 to 0.50 . From this result, to enhance a contribution of gyromagnetic spin rotation to complex permeability is effective for increasing $\mu^{\prime}$ in $\mathrm{GHz}$ range.

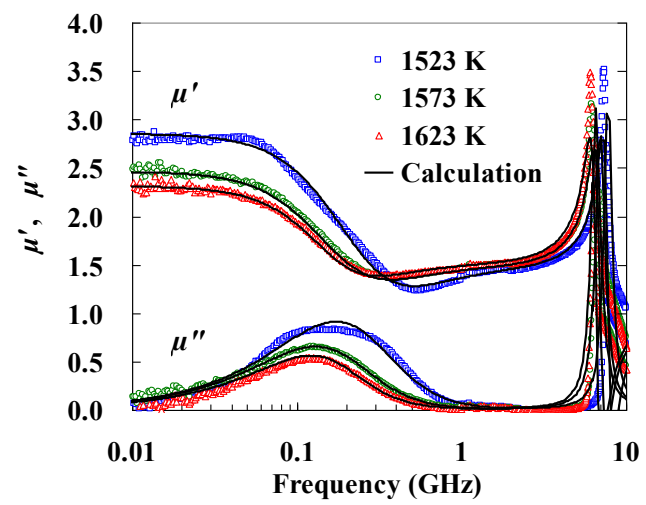

Fig. 2 Real part $\mu^{\prime}$ and imaginary part $\mu^{\prime \prime}$ of calculated and measured complex permeability spectra of $\mathrm{BaFe}_{8}\left(\mathrm{Ti}_{0.5} \mathrm{Mn}_{0.5}\right)_{4} \mathrm{O}_{19}$ with different sintering temperature.

Table II $\mu^{\prime}$ and $\tan \delta_{\mu}$ measured at $1.57 \mathrm{GHz}$ and parameters of calculated complex permeability of $\mathrm{BaFe}_{8}\left(\mathrm{Ti}_{0.5} \mathrm{Mn}_{0.5}\right)_{4} \mathrm{O}_{19}$.

\begin{tabular}{ccccccccc}
\hline \hline Sintering temperature $(\mathrm{K})$ & $\mu^{\prime}[1.57 \mathrm{GHz}]$ & $\tan \delta_{\mu}[1.57 \mathrm{GHz}]$ & $\chi_{\mathrm{s} 0}$ & $\omega_{\mathrm{s}}(\mathrm{GHz})$ & $\alpha$ & $\chi_{\mathrm{d} 0}$ & $\omega_{\mathrm{d}}(\mathrm{GHz})$ & $\boldsymbol{\beta}$ \\
\hline 1523 & $\mathbf{1 . 4 2}$ & $\mathbf{0 . 0 1 8}$ & $\mathbf{0 . 4 5}$ & 7.8 & $\mathbf{0 . 0 7}$ & $\mathbf{1 . 4 0}$ & $\mathbf{0 . 3 0}$ & $\mathbf{0 . 6 0}$ \\
1573 & 1.48 & 0.010 & 0.47 & 7.0 & $\mathbf{0 . 0 8}$ & $\mathbf{0 . 9 9}$ & $\mathbf{0 . 2 2}$ & $\mathbf{0 . 4 3}$ \\
1623 & 1.52 & 0.009 & $\mathbf{0 . 5 0}$ & $\mathbf{6 . 6}$ & $\mathbf{0 . 0 8}$ & $\mathbf{0 . 8 1}$ & $\mathbf{0 . 1 9}$ & $\mathbf{0 . 3 4}$ \\
\hline \hline
\end{tabular}




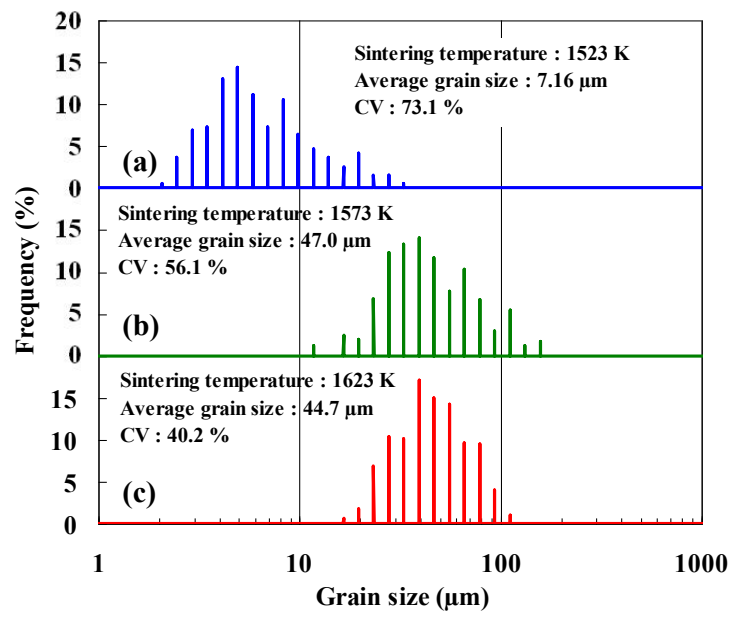

Fig. 3 Grain size distribution with average grain size and coefficient of variation (CV) of $\mathrm{BaFe}_{8}\left(\mathrm{Ti}_{0.5} \mathrm{Mn}_{0.5}\right)_{4} \mathrm{O}_{19}$ sintered separately at (a) $1523 \mathrm{~K}$, (b) $1573 \mathrm{~K}$, or (c) $1623 \mathrm{~K}$.

Effect of grain size on complex permeability in $\mathrm{GHz}$ range is also examined. Figure 3 shows distribution of grain size with average grain size and coefficient of variation (CV) of $\mathrm{BaFe}_{8}\left(\mathrm{Ti}_{0.5} \mathrm{Mn}_{0.5}\right)_{4} \mathrm{O}_{19}$. With increase of sintering temperature from $1523 \mathrm{~K}$ to $1623 \mathrm{~K}$, average grain size also increases from $7.16 \mu \mathrm{m}$ to $44.7 \mu \mathrm{m}$, whereas $\mathrm{CV}$ decreases from 73.1 to 40.2 indicating the distribution becomes narrower. When grain size becomes large and the variation decreases, lattice defects such as grain boundary and vacancy are supposed to decrease. Then net area of domain wall in a sample is decreased. Consequently, contribution of domain wall motion to permeability is decreased. On the other hand, contribution of gyromagnetic spin rotation to permeability is increased. And resonance frequency corresponding to the spin rotation shifts to lower frequency because of the decreased vacancy. A value of $\tan \delta_{\mu}$ at $1.57 \mathrm{GHz}$ decreases from 0.018 to 0.009 with the increase of grain size and the decrease of CV. Since a resonance peak corresponding to domain wall motion is shifted to lower frequency and become sharp by the altered grain size distribution, magnetic loss derived from skirts of the peak is reduced.

\section{CONCLUSION}

We investigated the effect of $\mathrm{Fe}^{3+}$ substitution and grain size on complex permeability of M-type hexaferrite $\mathrm{BaFe}_{12-x}\left(\mathrm{Ti}_{0.5} \mathrm{Mn}_{0.5}\right)_{x} \mathrm{O}_{19}(0 \leq x \leq 5)$. From the result of model fitting on the measured permeability taking account of a resonance of gyromagnetic spin rotation and that of domain wall motion, it was found that to enhance the contribution of the gyromagnetic spin rotation is effective for increase of $\mu^{\prime}$ in the $\mathrm{GHz}$ region. Furthermore, we compared grain size distribution with the complex permeability. It was shown that a resonance peak of domain wall shifts to lower frequency and shape of the peak becomes narrower because of the enlarged grain and the decreased size variation, indicating effectiveness to reduce magnetic loss in the GHz region. Our study shows M-type hexaferrites could be candidates for a magnetic material used in high frequency region with both low magnetic loss and high $\mu^{\prime}$. However, further increased value of $\mu^{\prime}$ is required for applying M-type hexaferrites to devices used in the $\mathrm{GHz}$ region.

\section{REFERENCES}

[1] Y. Shirakata, N. Hidaka, M. Ishitsuka, A. Teramoto, and T. Ohmi, IEEE Trans. Magn. 44, 2100 (2008).

[2] K. Kakizaki, N. Hiratsuka, and T. Namikawa, J. Magn. Magn, Mater. 176, 36 (1997).

[3] S. Sugimoto, K.Okayama, H. Ota, M. Kimura, Y. Yoshida, H. Nakamura, et al., J. Magn. Soc. Jpn. 23, 611 (1999).

[4] J. Lee, Y. Hong, J. Park, W. Seong, G. Kim, and A. Morisako, IEEE Magn. Lett. 2, 5000204 (2011).

[5] Takanori Tsutaoka, J. Appl. Phys. 93, 2789 (2003). 